\title{
Dose-Response Outcomes Associated with Different Forms of Locomotor Training in Persons with Chronic Motor-Incomplete Spinal Cord Injury
}

\author{
Evan B. Sandler, Kathryn E. Roach, and Edelle C. Field-Fote ${ }^{1,3,4}$
}

\begin{abstract}
Outcomes of training are thought to be related to the amount of training (training dose). Although various approaches to locomotor training have been used to improve walking function in persons with spinal cord injury (SCI), little is known about the relationship between dose of locomotor training and walking outcomes. This secondary analysis aimed to identify the relationship between training dose and improvement in walking distance and speed associated with locomotor training in participants with chronic motor-incomplete spinal cord injury (MISCI). We compared the dose-response relationships associated with each of four different locomotor training approaches. Participants were randomized to either: treadmillbased training with manual assistance $(\mathrm{TM}=17)$, treadmill-based training with stimulation $(\mathrm{TS}=18)$, overground training with stimulation $(\mathrm{OG}=15)$, and treadmill-based training with locomotor robotic device assistance $(\mathrm{LR}=14)$. Subjects trained 5 days/week for 12 weeks, with a target of 60 training sessions. The distance-dose and time-dose were calculated based on the total distance and total time, respectively, participants engaged in walking over all sessions combined. Primary outcome measures included walking distance (traversed in $2 \mathrm{~min}$ ) and walking speed (over $10 \mathrm{~m}$ ). Only OG training showed a good correlation between distance-dose and change in walking distance and speed walked over ground $(r=0.61, p=0.02$; $r=0.62, p=0.01)$. None of the treadmill-based training approaches were associated with significant correlations between training dose and improvement of functional walking outcome. The findings suggest that greater distance achieved over the course of OG training is associated with better walking outcomes in the studied population. Further investigation to identify the essential elements that determine outcomes would be valuable for guiding rehabilitation.
\end{abstract}

Keywords: distance; OG training; rehabilitation; walking speed

\section{Introduction}

D ESPITE THE LARGE NUMBER OF STUDIES related to cellular and pharmacological therapies for improving motor function after spinal cord injury (SCI), outcomes associated with these approaches are most robust when combined with rehabilitation. ${ }^{1}$ However, although dose-response relationships are almost universally considered to be a component of intervention development in cellular and pharmacological therapies, there have been few studies of dose-response relationships in physical therapeutic interventions. $^{2}$ Thus far, dose has been described in task-related training with a "more is better" approach. ${ }^{3-5}$ Studies of real-world clinical practice, however, show the actual dose of training received is low ${ }^{5}$ compared with that shown by animal studies to be necessary to produce change in motor function.

Rehabilitation after SCI aims to increase functional mobility and improve quality of life. At 1 year post-injury, $\sim 41 \%$ of persons with SCI are classified as having chronic motor-incomplete SCI (MISCI). ${ }^{6}$ Many individuals with MISCI have the potential to regain some walking function. ${ }^{7-11}$ Locomotor training with partial support for body weight has been extensively studied in recent years. This training can be effectively performed in either the overground (OG) or the treadmill environment. Beyond differences in training environment, different approaches can be used to provide assistance for stepping, and studies wherein assistance was provided by therapists, functional electrical stimulation (FES), or robotic devices have been published.

Of the many locomotor training studies that have included participants with SCI, few have compared outcomes among different training approaches. ${ }^{7,9,12-18}$ For the outcome of walking speed, these studies have identified no single intervention approach as being significantly better in comparison with others. ${ }^{7,9,12-18}$ However, for the outcome of walking distance, the largest randomized clinical trial in persons with chronic MISCI identified significantly

\footnotetext{
${ }^{1}$ Crawford Research Institute, Shepherd Center, Atlanta, Georgia.

${ }^{2}$ Department of Physical Therapy, and ${ }^{4}$ The Miami Project to Cure Paralysis, Miller School of Medicine, University of Miami, Miami, Florida.

${ }^{3}$ Department of Rehabilitation Medicine, School of Medicine, Emory University, Atlanta, Georgia.
} 
greater gains in walking distance following OG training compared with gains achieved with three treadmill-based training approaches with different types of assistance for stepping. ${ }^{19}$ Moreover, the OG training approach had the largest effect size for outcomes related to OG walking distance and speed compared with treadmill-based training approaches. ${ }^{19}$

Aside from the numerous types of interventions that have been studied with the goal of improving walking function in persons with SCI, there is also a wide range of training protocols in the locomotor training literature. Measures that represent training dose within these protocols may include number of steps taken, treadmill training speed, distance walked, time walked (within a single treatment session and cumulative), and total number of treatment sessions. ${ }^{3}$ Each of these parameters represents aspects of training dose, and studying each in isolation may contribute to understanding the effect of dose on locomotor training outcomes. Reported values for number of training sessions range from 17 to 251 , with training session durations ranging from 20 to 90 min. $^{12,13,15,20-25}$ Parameters such as distance and time walked per session or cumulatively over multiple sessions have either not been reported, or if reported, have not been analyzed for their possible influence on outcomes.

Only one previous study has reported dose-related data. ${ }^{15}$ In that study, dose was reported in terms of mean walking distance traversed over all sessions for three different interventions, but the number of training sessions (24) and sample size per group $(n=10)$ of the study were small, and no statistical analysis was performed to assess the relationship between this measure of dose and the observed walking outcomes. ${ }^{15}$ There are no prior studies that have compared the relationships between training dose and functional walking outcomes in persons with MISCI across different locomotor training approaches.

There is good evidence to indicate that outcomes of rehabilitation are related to amount or "dose" of the rehabilitation intervention. Understanding the dose-response relationship in locomotor training is necessary to identify the approaches that are associated with the greatest improvement with the least expenditure of time and resources. The purpose of this secondary analysis was to identify the relationship between locomotor training dose and outcomes of walking distance and speed for each of four locomotor training approaches in participants with chronic MISCI. This report represents the first direct comparison of dose-response relationships of multiple available interventions aimed at improving walking function in persons with MISCI.

\section{Methods}

Complete details of the study methods are described in a prior publication, including training protocol and the Consolidated Standards of Reporting Trials (CONSORT) diagram outlining enrollment, allocation, randomization, follow-up, and analysis. ${ }^{19}$

\section{Design overview}

This study is a secondary analysis of a single-blind, randomized clinical trial to compare four locomotor training interventions for individuals with chronic ( $\geq 1$ year) MISCI.

\section{Settings and participants}

This study was conducted at the Miami Project to Cure Paralysis, University of Miami Miller School of Medicine. Inclusion criteria were: injury classification of American Spinal Cord Association (ASIA) Impairment Scale (AIS) C or D, injury level $\geq \mathrm{T} 10$, ability to take at least one step with one leg, and ability to rise to a standing position with no more than moderate assistance (50\% effort) from one other person. ${ }^{26}$ Exclusion criteria were: current orthopedic problems, history of cardiac condition, or radiographic evidence of hip pathology that could be aggravated by training. Participants gave written and oral informed consent according to the protocol of a study approved by Human Studies Research Office, Miller School of Medicine, University of Miami.

\section{Interventions}

According to the protocol of the primary study, participants were randomized into one of four intervention groups: 1) treadmill-based training with manual assistance for stepping provided by trainer(s) (TM), 2) treadmill-based training with electrical stimulation of the common peroneal nerve to elicit a flexion withdrawal response at the onset of swing phase (TS), 3) OG training with functional electrical stimulation to assist with dorsiflexion during swing phase (OG), and 4) treadmill-based training with a robotic gait orthosis (using 100\% guidance force) (LR). Techniques applied to interventions requiring assistance have been previously published. ${ }^{27}$ Training was performed 5 days per week for 12 weeks with a target of 60 sessions completed. Allotted time for each training session was $60 \mathrm{~min}$.

\section{Outcomes}

Measurements of walking distance (traversed in $2 \mathrm{~min}$ ) and walking speed (over $10 \mathrm{~m}$ ) were acquired prior to and following training. A training log was maintained for each subject; for each session the distance walked and the time walked was recorded. For subjects trained using a treadmill-based approach, the treadmill speed during training was also recorded.

\section{Statistical analysis}

All data analyses were performed in Excel (Microsoft, Redmond, WA) and SAS version 9.4 (SAS Institue Inc., Cary, NC). For each participant, distance-dose was calculated by summing distance walked for all sessions; time-dose was calculated by summing time walked for all sessions (time spent resting between walking bouts was not included in the time-dose calculation).

Within each group, Pearson correlations were calculated to examine 1) the relationships between distance-dose and trainingrelated improvements in walking distance and walking speed, 2) the relationships between time-dose and training-related improvements in walking distance and speed, and 3) the relationship between distance-dose and time-dose.

\section{Results}

Sixty-four participants completed all training and testing. Among the four training groups, data were equivalent at baseline for the following variables: age, height, weight, sex, race, Lower Extremity Motor Score (LEMS) total, walking distance, and walking speed. ${ }^{19}$ Mean walking speeds for the OG, TS, TM, and LR groups were $0.19 \mathrm{~m} / \mathrm{sec}, 0.18 \mathrm{~m} / \mathrm{sec}, 0.17 \mathrm{~m} / \mathrm{sec}$, and $0.17 \mathrm{~m} / \mathrm{sec}$, respectively. Distance walked in $2 \mathrm{~min}$ averaged $24.0 \mathrm{~m}, 20.6 \mathrm{~m}, 22.1 \mathrm{~m}$, and $16.8 \mathrm{~m}$ for the OG, TS, TM, and LR groups, respectively. ${ }^{19}$

Across all groups, participants completed a mean of 49 (SD, 7; range, 27-58) sessions. Mean values of distance-dose in meters were $\mathrm{OG}=2989$, $\mathrm{TS}=1141$, $\mathrm{TM}=1700$, and $\mathrm{LR}=14793$. Mean values of time-dose in minutes were $\mathrm{OG}=176.71$, $\mathrm{TS}=60.58$, $\mathrm{TM}=70.93$, and $\mathrm{LR}=358.26$ (See Fig. 1).

Correlations between distance-dose and change in walking distance ranged from $r=-0.23$ to $r=0.61$. The OG group was the only group for which the relationship between distance-dose and change 


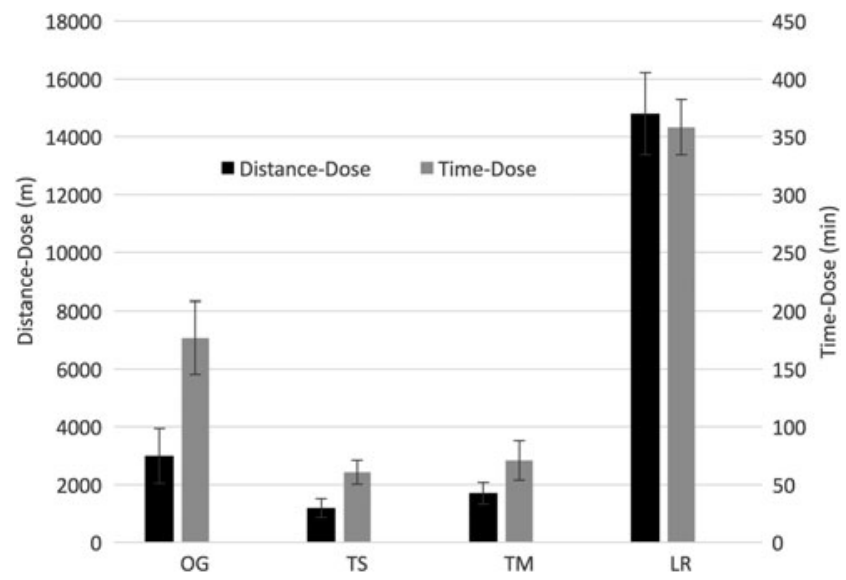

FIG. 1. Distance-dose (m) and time-dose (min) means by group. Data were calculated by summing the distance traversed across all sessions of training and time spent walking during all sessions of training. Error bars indicate standard error of the mean. Distance-dose means $( \pm$ SE): OG $=2989( \pm 951), \mathrm{TS}=1141( \pm 302)$, $\mathrm{TM}=1700( \pm 381), \mathrm{LR}=14793( \pm 1418)$. Time-dose means $( \pm \mathrm{SE})$ : $\mathrm{OG}=176.71( \pm 31.41), \mathrm{TS}=60.58( \pm 10.45), \mathrm{TM}=70.93( \pm 17.06)$, $\mathrm{LR}=358.26( \pm 23.99)$.

in walking distance was significant $(r=0.61, p=0.02)$. Correlations between distance-dose and change in walking speed ranged from $r=-0.18$ to $r=0.62$. The OG group was the only group for which the relationship between distance-dose and change in walking speed was significant $(r=0.62, p=0.01)$. Only low, nonsignificant correlations were found in the relationships between time-dose and change in walking distance $(r=-0.12-0.25)$, and between timedose and walking speed $(r=-0.25$ to -0.09 ) (See Table 1 and Fig. 2).

Correlations between distance-dose and time-dose within each training group were also assessed. All treadmill-based training groups had strong and significant correlations between distancedose and time-dose ( $r=0.80[\mathrm{TS}], 0.92[\mathrm{TM}]$, and 0.95[LR], $p<0.0001)$. For the OG group the correlation was low and nonsignificant $(r=0.39, p=0.15)$.

\section{Discussion}

The goal of the present study was to characterize, for four different approaches to locomotor training used in persons with chronic MISCI, how the distance walked and time walked during training relates to improvements in the outcome measures of OG walking distance and OG walking speed. Results indicated that, for distance-dose, greater distance walked over the course of OG

Table 1. Correlations between Dose and Walking Distance and Speed Outcomes

\begin{tabular}{lccccc} 
& \multicolumn{2}{c}{ Distance-Dose } & & \multicolumn{2}{c}{ Time-Dose } \\
\cline { 2 - 3 } \cline { 6 - 6 } & Distance & Speed & & Distance & Speed \\
\hline OG & $0.61^{*}$ & $0.62^{\dagger}$ & & -0.10 & -0.09 \\
TS & 0.30 & -0.15 & & 0.25 & -0.25 \\
TM & -0.23 & -0.18 & & -0.12 & -0.13 \\
LR & -0.12 & 0.01 & & -0.12 & -0.13 \\
\hline
\end{tabular}

$* p=0.016 ;{ }^{\dagger} p=0.013$ training was associated with greater increases in both distance- and speed-related outcome measures (distance walked in $2 \mathrm{~min}$ and OG walking speed). For the treadmill-based training approaches, distance-dose was not significantly related to change in either walking distance or walking speed. For time-dose, more time walking during training was not significantly related to change in outcomes for either speed or distance for any of the four training approaches.

Even though a uniform training protocol, in terms of time allocated to training each session, was shared among all groups, the resulting distance-dose and time-dose varied widely among the four different training approaches. When training-related variables were summed across all sessions, the LR group achieved a distancedose that was more than 10 times greater than that of TS group, almost 10 times greater than that of the TM group, and 5 times greater than that of the OG group. Similarly, the OG group traversed more than twice the distance during training as did the TS group, and almost twice the distance of the TM group. The timedose for the LR group was six and five times greater than that of the TS and TM groups, respectively, and two times greater than that of the OG group. The time-dose of the OG training group was almost 3 times the time-dose of the TS group and 2.5 times that of the TM group.

There was a meaningful relationship between distance-dose and functional improvements for the OG approach, whereas there was no such relationship for the LR, TM, or TS approaches. It would be valuable to consider factors that may have contributed to these differences. Data related to gait quality from this same study indicated that cadence and step length of both the stronger and weaker leg increased across all groups. ${ }^{28}$ It is likely that an increased number of steps taken results in a greater distance traversed. In turn, the results of this study suggest that greater number of step repetitions is associated with greater functional change in walking ability, and the effect is most robust when the training is performed under conditions that most closely represent the real-world environment.

To our knowledge, the only prior study that assessed doserelated measures of locomotor training in participants with SCI compared robotic and manually assisted groups trained on a treadmill with body weight support, and a group that trained over ground without body weight support. The distance-dose for the groups that trained on the treadmill were, on average, more than twice that of the group that trained over ground. Despite this, the outcomes were comparable for all the groups, with no significant between-group differences in change of gait speed and distance. ${ }^{15}$ The group that trained over ground in the aforementioned study completed, over the course of the study, an average of $1282 \mathrm{~m}$ compared with the $2989 \mathrm{~m}$ of the current study. As we found a strong and significant correlation between distance-dose and change in walking outcomes associated with OG training, it is plausible that the dose of the OG training in the previous study was not sufficient to achieve between-group differences in dose-related effects.

For the variable of time-dose, none of the training groups achieved significant correlations between time-dose and change in walking outcomes. This suggests that it is not the time spent in training, but rather how the individual engages in the activity during that time that is key to improving performance. This point is clear in the LR group, which had the largest overall training doses (both distance-dose and time-dose), but demonstrated the poorest relationship between training dose and outcomes; that is, highest training doses with the smallest gains in walking distance and speed. As has been described previously, ${ }^{19}$ the LR training was 

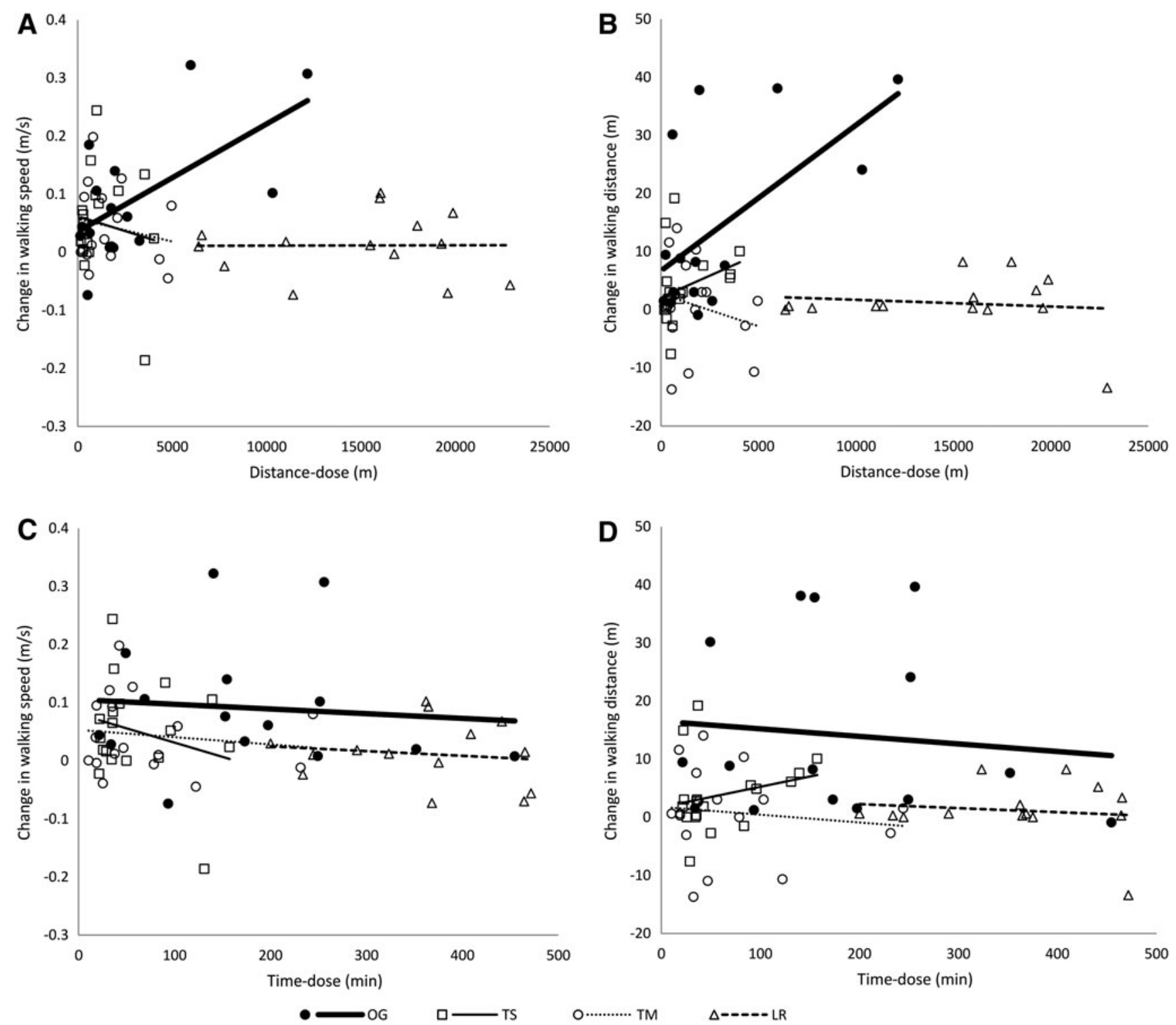

FIG. 2. Walking-related outcomes as a function of training dose for each subject. (A) Change in walking speed as a function of distance-dose. (B) Change in walking distance as a function of distance-dose. (C) Change in walking speed as a function of time-dose. (D) Change in walking distance as a function of time-dose. Trend lines indicate the relationship between variables for each of the four training approaches. Correlation coefficients $(r)$ and significance can be found in Table 1.

performed with $100 \%$ guidance forces, which suggests that providing only the afferent input associated with movement does not promote the ability to volitionally produce that movement, and also argues for the need for volitional effort to realize a training effect.

The correlation between distance-dose and time-dose was strong for all treadmill-based interventions, but was not for the OG intervention. This result may be anticipated, given that all participants trained over ground were urged to walk at their maximum comfortable walking speed, but for the treadmill-based training groups, the speed was controlled by the trainer based on a standardized protocol for progressing walking speed. ${ }^{19}$ Among participants in the OG group, individual differences in self-selected stepping behavior during training resulted in great variability of distance covered per given time, most often a shorter distance compared with that for participants in the three treadmill-based training groups. It is possible that persons training over ground select speeds that they can sustain for longer periods of time, regardless of en- couragement to walk faster. Conversely, for persons training on the treadmill, wherein speed is selected by the trainer, individuals are required to walk at a faster speed, which they can sustain for only a short duration. At baseline for the current study population, the average walking speed across all groups was $<0.2 \mathrm{~m} / \mathrm{sec}$ and average lower extremity motor scores were $<15$ for both lower extremities. ${ }^{19}$ In persons with SCI the ability to elicit volitional contractions is decreased proportionally to the impairment, especially speed of contraction. ${ }^{29,30}$ Subsequently, there is evidence that in persons with MISCI, muscle activity is more optimal at lower speeds resembling self-selected speed. ${ }^{31}$ In contrast, a recent study demonstrated that muscle activity increased as training intensity (that is, speed) increased. ${ }^{32}$ However, walking was tested only on the treadmill, and therefore it is not known whether this translates to improvements in real-world overground walking. In addition, the participants in that study were able to achieve walking speeds of at least $0.3 \mathrm{~m} / \mathrm{sec}$ without physical assistance at baseline, indicating they were higher 
functioning than subjects in our study. The results of our study seem to indicate that emphasizing speed during training is not the optimal approach if the goal is to increase overground walking ability. Furthermore, the two contributing variables to speed, distance and time, are not interchangeable as measures of dose in training protocols.

\section{Limitations}

This secondary analysis has several limitations. First, we did not analyze the distribution of dose-related variables over the course of training. For example, it is possible that individual participants achieved similar distance-dose (or time-dose) over different total numbers of sessions. For this reason, we cannot rule out the possible influence of completing a higher volume of training within a shorter period of time (i.e., massed practice vs. distributed practice). Second, although the percentage of body weight support provided did not exceed $30 \%$ in any of the groups after the first week of training, the percent body weight support was adjusted on an individual basis during each training session, and this may have impacted the distance-dose and time-dose achieved. It is also possible that individual differences in tolerance to each intervention resulted in differences in dose-related variables.

\section{Conclusions}

Our study examined the correlations between distance-dose and time-dose and change in functional overground walking outcomes. Total distance walked during training over all sessions is directly correlated to change in OG walking speed and distance in individuals whose locomotor training involved OG training with body weight support and FES to assist with dorsiflexion. This finding implies that a greater walking distance during OG training yields better walking function in the studied population. In the treadmillbased training interventions, relationships between training dose (both time and distance) and walking outcomes were poor. Distance-dose and time-dose were strongly correlated among all treadmill-based interventions, whereas there was poor correlation between these two parameters in overground training. Identification of appropriate measures of dose for a given intervention should be considered in protocol design. Future research protocols for locomotor training of individuals with chronic MISCI should incorporate distribution of dose over training in order to identify effective utilization of therapeutic resources.

\section{Acknowledgments}

This study was funded by National Institutes of Health grant R01HD41487 (to Dr. Field-Fote) and The Miami Project to Cure Paralysis. We thank Stephanie Hiser and Lauren Stewart for their assistance and support.

Clinical Trial Registry Number: NCT01095380

\section{Author Disclosure Statement}

No competing financial interests exist.

\section{References}

1. Gomes-Osman, J., Cortes, M., Guest, J., and Pascual-Leone, A. (2016). A systematic review of experimental strategies aimed at improving motor function after acute and chronic spinal cord injury. J. Neurotrauma 33, 425-438.

2. Dobkin, B.H. (2005). Rehabilitation and functional neuroimaging dose-response trajectories for clinical trials. Neurorehabil. Neural Repair 19, 276-282.
3. Lang, C.E., MacDonald, J.R., and Gnip, C. (2007). Counting repetitions: an observational study of outpatient therapy for people with hemiparesis post-stroke. J. Neurol. Phys. Ther. 31, 3-10.

4. Lang, C.E., Lohse, K.R., and Birkenmeier, R.L. (2015). Dose and timing in neurorehabilitation. Curr. Opin. Neurol. 28, 549-555.

5. Zbogar, D., Eng, J.J., Miller, W.C., Krassioukov, A.V., and Verrier, M.C. (2016). Movement repititions in physical and occupational therapy during spinal cord injury rehabilitation. Spinal Cord DOI: 10.1038/sc.2016.129. [Epub ahead of print.]

6. Center, N.S.C.I.S. (2014). 2014 Annual Report Complete Public Version.

7. Alexeeva, N., Sames, C., Jacobs, P.L., Hobday, L., Distasio, M.M., Mitchell, S. A, and Calancie, B. (2011). Comparison of training methods to improve walking in persons with chronic spinal cord injury: a randomized clinical trial. J. Spinal Cord Med. 34, 362-379.

8. Benito-Penalva, J., Edwards, D.J., Opisso, E., Cortes, M., LopezBlazquez, R., Murillo, N., Costa, U., Tormos, J.M., Vidal-Samsó, J., Valls-Solé, J., European Multicenter Study about Human Spinal Cord Injury Study Group, and Medina, J. (2012). Gait training in human spinal cord injury using electromechanical systems: effect of device type and patient characteristics. Arch. Phys. Med. Rehabil. 93, 404412.

9. Esclarín-Ruz, A., Alcobendas-Maestro, M., Casado-Lopez, R., PerezMateos, G., Florido-Sanchez, M.A., Gonzalez-Valdizan, E., and Martin, J.L.R. (2014). A comparison of robotic walking therapy and conventional walking therapy in individuals with upper versus lower motor neuron lesions: a randomized controlled trial. Arch. Phys. Med. Rehabil. 95, 1023-1031.

10. Wu, M., Landry, J.M., Schmit, B.D., Hornby, T.G., and Yen, S.C. (2012). Robotic resistance treadmill training improves locomotor function in human spinal cord injury: a pilot study. Arch. Phys. Med. Rehabil. 93, 782-789.

11. Musselman, K.E., Fouad, K., Misiaszek, J.E., and Yang, J.F. (2009). Training of walking skills overground and on the treadmill: case series on individuals with incomplete spinal cord injury. Phys. Ther. 89, 601-611.

12. Alcobendas-Maestro, M., Esclarín-Ruz, A., Casado-López, R.M., Muñoz-González, A., Pérez-Mateos, G., González-Valdizán, E., and Martín, J.L.R. (2012). Lokomat robotic-assisted versus overground training within 3 to 6 months of incomplete spinal cord lesion: randomized controlled trial. Neurorehabil. Neural Repair 26, 1058-1063.

13. Dobkin, B., Apple, D., Barbeau, H., Basso, M., Behrman, A., Deforge, D., Ditunno, J., Dudley, G., Elashoff, R., Fugate, L., Harkema, S., Saulino, M., Scott, M., and Spinal Cord Injury Locomotor Trial Group. (2006). Weight-supported treadmill vs over-ground training for walking after acute incomplete SCI. Neurology 66, 484-493.

14. Duffell, L.D., Brown, G.L., and Mirbagheri, M.M. (2015). Interventions to reduce spasticity and improve function in people with chronic incomplete spinal cord injury: distinctions revealed by different analytical methods. Neurorehabil. Neural Repair 29, 566-576.

15. Hornby, G., Campbell, D., Zemon, D., and Kahn, J. (2005). Clinical and quantitative evaluation of robotic-assisted treadmill walking to retrain ambulation after spinal cord injury. Top. Spinal Cord Inj. Rehabil. 11, 1-17.

16. Kapadia, N., Masani, K., Catharine Craven, B., Giangregorio, L.M., Hitzig, S.L., Richards, K., and Popovic, M.R. (2014). A randomized trial of functional electrical stimulation for walking in incomplete spinal cord injury: effects on walking competency. J. Spinal Cord Med. 37, 511-524.

17. Labruyère, R., and van Hedel, H.J.A. (2014). Strength training versus robot-assisted gait training after incomplete spinal cord injury: a randomized pilot study in patients depending on walking assistance. J. Neuroeng. Rehabil. 11, 4.

18. Sharp, K.G., Gramer, R., Butler, L., Cramer, S.C., Hade, E., and Page, S.J. (2014). Effect of overground training augmented by mental practice on gait velocity in chronic, incomplete spinal cord injury. Arch. Phys. Med. Rehabil. 95, 615-621.

19. Field-Fote, E.C., and Roach, K.E. (2011). Influence of a locomotor training approach on walking speed and distance in people with chronic spinal cord injury: a randomized clinical trial. Phys. Ther. 91, 48-60.

20. Harkema, S.J., Schmidt-Read, M., Lorenz, D.J., Edgerton, V.R., and Behrman, A.L. (2012). Balance and ambulation improvements in individuals with chronic incomplete spinal cord injury using locomotor training-based rehabilitation. Arch. Phys. Med. Rehabil. 93, 15081517. 
21. Wirz, M., Zemon, D.H., Rupp, R., Scheel, A., Colombo, G., Dietz, V., and Hornby, T.G. (2005). Effectiveness of automated locomotor training in patients with chronic incomplete spinal cord injury: a multicenter trial. Arch. Phys. Med. Rehabil. 86, 672-680.

22. Winchester, P., McColl, R., Querry, R., Foreman, N., Mosby, J., Tansey, K., and Williamson, J. (2005). Changes in supraspinal activation patterns following robotic locomotor therapy in motor-incomplete spinal cord injury. Neurorehabil. Neural Repair 19, 313-324.

23. Postans, N.J., Hasler, J.P., Granat, M.H., and Maxwell, D.J. (2004). Functional electric stimulation to augment partial weight-bearing supported treadmill training for patients with acute incomplete spinal cord injury: A pilot study. Arch. Phys. Med. Rehabil. 85 , 604-610.

24. Thrasher, T.A., Flett, H.M., and Popovic, M.R. (2006). Gait training regimen for incomplete spinal cord injury using functional electrical stimulation. Spinal Cord 44, 357-361.

25. Field-Fote, E.C. (2001). Combined use of body weight support, functional electric stimulation, and treadmill training to improve walking ability in individuals with chronic incomplete spinal cord injury. Arch. Phys. Med. Rehabil. 82, 818-824.

26. Marino, R.J., Barros, T., Biering-Sorensen, F., Burns, S.P., Donovan, W.H., Graves, D.E., Haak, M., Hudson, L.M., Priebe, M.M., and ASIA Neurological Standards Committee 2002. (2003). International standards for neurological classification of spinal cord injury. J. Spinal Cord Med. 26, Suppl. 1, S50-66.

27. Field-Fote, E.C., Lindley, S.D., and Sherman, A.L. (2005). Locomotor training approaches for individuals with spinal cord injury: a preliminary report of walking-related outcomes. J. Neurol. Phys. Ther. 29, 127-137.
28. Nooijen, C.F.J., Ter Hoeve, N., and Field-Fote, E.C. (2009). Gait quality is improved by locomotor training in individuals with SCI regardless of training approach. J. Neuroeng. Rehabil. 6, 36.

29. Petersen, J.A., Spiess, M., Curt, A., Dietz, V., Schubert, M., and EMSCI Study Group. (2012). Spinal cord injury: one-year evolution of motor-evoked potentials and recovery of leg motor function in 255 patients. Neurorehabil. Neural Repair 26, 939-948.

30. Wirth, B., Van Hedel, H.J.A., and Curt, A. (2008). Changes in corticospinal function and ankle motor control during recovery from incomplete spinal cord injury. J. Neurotrauma 25, 467-478.

31. Meyns, P., Van de Crommert, H.W.A.A., Rijken, H., van Kuppevelt, D.H.J.M., and Duysens, J. (2014). Locomotor training with body weight support in SCI: EMG improvement is more optimally expressed at a low testing speed. Spinal Cord 52, 887-893.

32. Leech, K.A., Kinnaird, C.R., Holleran, C.L., Kahn, J., and Hornby, T.G. (2016). Effects of locomotor exercise intensity on gait performance in individuals with incomplete spinal cord injury. Phys. Ther. 96, 1919-1929.

Address correspondence to: Edelle C. Field-Fote, PT, PhD Crawford Research Institute Shepherd Center 2020 Peachtree Road NW Atlanta, GA 30309

E-mail: edelle_field-fote@ shepherd.org 